\title{
An Evaluation of Human Immunodeficiency Virus Pathogenicity and Treatment Using Glycobiology
}

\author{
Aleksandra Leligdowicz, B.Sc. *.
}

\section{INTRODUCTION}

Over 50 million people are infected with the Human Immunodeficiency Virus (HIV) worldwide (1). Caring for the millions of people living with HIV and AIDS is essential. Over 40 therapies are approved by the Food and Drug Administration and as a result, the death rate has fallen dramatically across Europe and the United States. However, AIDS is increasingly a disease of the poor and medicines are where the problem is not, while the problem is where the medicines are not (2). Terminating the HIV epidemic is of utmost significance especially to developing countries, which account for over $95 \%$ of new HIV infections (1). Therefore, controlling the virus is important not only to the state of world health, but also to global development.

Glycobiology, the study of carbohydrates, is an interesting topic to medical research. Critical biological processes, including regulation of the growth and mobility of cells, immune responses, and responses of cells to hormones and growth factors, all depend on carbohydrates. In addition, viruses, including HIV, use cell-surface carbohydrates to get into cells and initiate infections. The aim of this review is to demonstrate how the expanding research in glycobiology can be applied to understanding the structure of HIV, as well as developing potential treatment approaches.

\section{ESSENTIALS OF GLYCOBIOLOGY}

One of the post-translational modifications that occurs on proteins after they have begun to be translated

\footnotetext{
* To whom correspondence should be addressed: 418 Ave des Pins Ouest, Apt 34, Montreal, Quebec H2W 1S2, Canada.

E-mail address: alelig@ po-box.mcgill.ca.
}

in the endoplasmic reticulum (ER) is N-linked addition of carbohydrate chains, resulting in the formation of glycoproteins (figure 1)

Glycoproteins contain one or more carbohydrate residues. The carbohydrate moieties of glycoproteins help to determine the tertiary structure of a protein. In addition, they may serve as biological labels, marking the proteins for different fates. This is possible because the number of possible permutations and combinations of monosaccharide types and glycosidic linkages in an oligosaccharide is astronomical (4). Each oligosaccharide can therefore present a different face that can be recognized by not only specific enzymes but more importantly for HIV infection, by specific receptors.

\section{OVERVIEW OF HIV INFECTION}

The human immunodeficiency virus can be classified as types one (HIV-1) and two (HIV-2). HIV-1 is older, more infectious, and accounts for the vast majority of HIV worldwide while HIV-2 is present mainly in Western Africa (5). Since HIV -1 accounts for the majority of the infections worldwide and since it is 3.55 -fold more infectious than HIV-2, it will be the virus type of focus of this review.

HIV-1 is the causative agent for the acquired immunodeficiency syndrome, or AIDS. Infection begins when HIV interacts with a host cell surface membrane protein (CD4) via a viral envelope glycoprotein. The glycoprotein is an oligomer of extracellular (gp120) and transmembrane (gp41) glycoproteins (6). The gp120 is responsible for virion binding to CD4 receptors of host cells, whereas gp41 mediates fusion of the HIV virus and the host cell membrane. The fusion of membranes allows the viral 


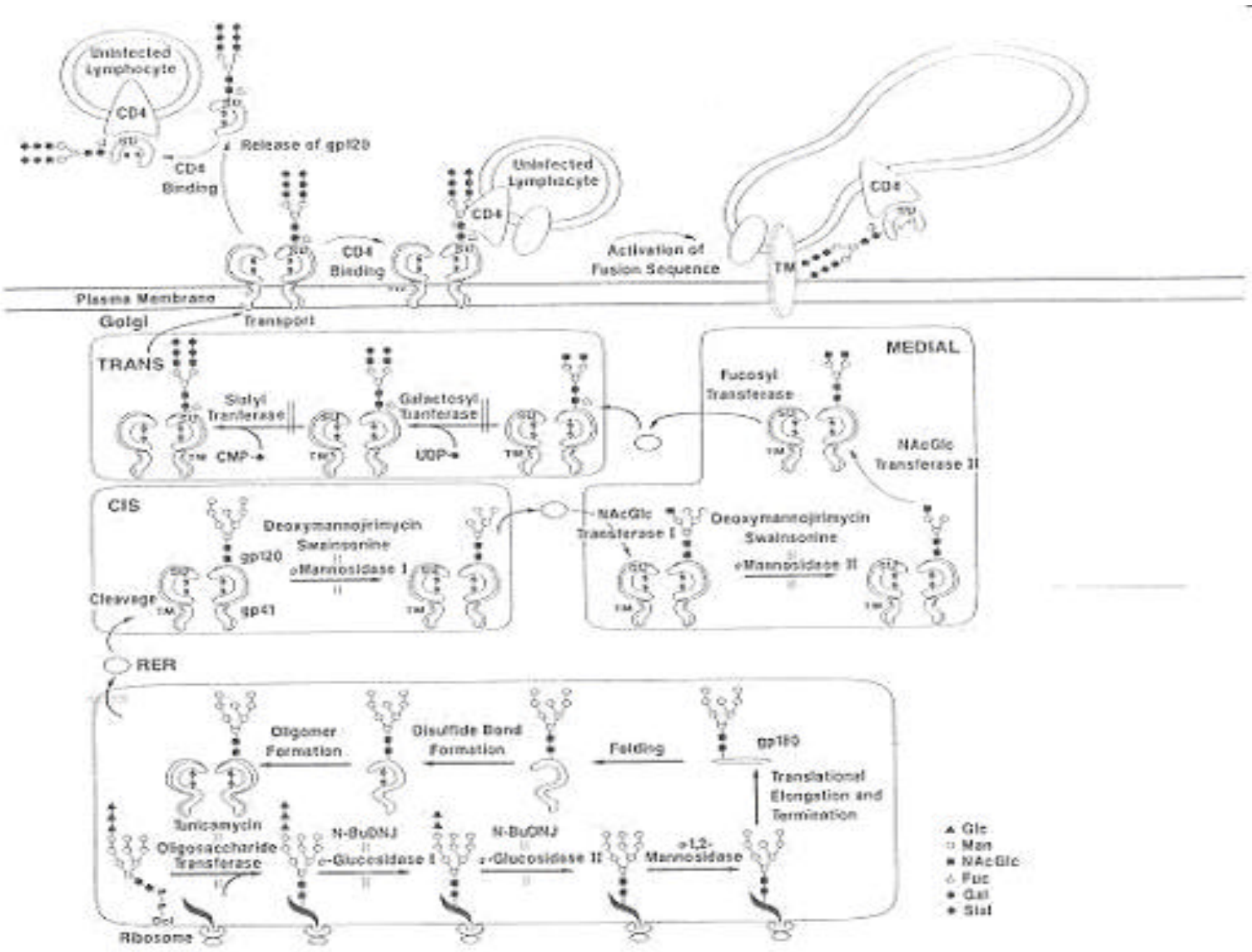

Figure 1. Steps in the formation of N-linked oligosaccharides in the lumen of the rough endoplasmic reticulum ER and the various compartments of the Golgi complex. The attachment of oligosaccharides is via an N-glycosidic bond to the side chain of an asparagine residue on the protein. The asparagine is part of the sequon Asn-X-Thr/Ser. The sequon is recognized by an enzyme that transfers the oligosaccharide from dolichol to Asn in the peptide. The first seven sugars ( 2 GlcNAc's and 5 Mannoses), are transferred one at a time to the dolichol-PP on the cytosolic side of ER. The oligosaccharide then flips across the ER membrane and the rest of the monosaccharides are added to the glycan on the luminal side of the membrane. After the oligosaccharide is assembled, it is transferred to an asparagnine residue on the nascent peptide. Once the glycan is attached, it undergoes removal steps mediated by glucosidases (glucose cleaving enzymes) that take off the terminal glucose residues. The protein is then shipped to the Golgi complex, where most of the terminal mannose units are removed by mannosidases and a variety of sugars.

Various Glycosylation pathway inhibitors are listed above the enzymes associated with the steps in the pathway (3).

particle to enter the cell by receptor-mediated endocytosis. Once the virus is inside the cell, it releases its RNA genome and the enzyme reverse transcriptase (RT). RT reverse transcribes the viral RNA into DNA. The HIV DNA moves into the nucleus where it is inserted into the host cell's genome. New HIV virions are made by the host cell, which replicates and transcribes the HIV DNA, and translates the viral transcript as well. Newly formed virions are released from the host cell, ready to infect the next CD4+ host cell (T helper-cell).

The precursor to gp120 and gp41 is gp160. This glycoprotein is heavily glycosylated in the ER lumen, where disulfide formation also occurs. The gp160 is cleaved intracellularly by a host protease in the late Golgi compartment to yield the noncovalently linked gp120 and gp41 complexes (figure 2 a, b, c), which are then transported to the host plasma membrane. At the surface, the complexes can be used by the emerging virus when it buds out from the cell to equip the virus for infection of new CD4 cells. In addition, the gp120gp41 complex in the host plasma membrane can bind a CD4 receptor on an uninfected cell, resulting in cell fusion (6).

\section{HIV GLYCOPROTEIN STRUCTURE}

The use of mutated recombinant HIV glycoproteins to study their structure

The structure of the HIV envelope glycoproteins (gp120 and gp41) is important for developing drugs that can function to eradicate the virus. An understanding of the chemical nature and the processing of the oligosaccharide chains attached to the envelope proteins, and the identification of the sites where posttranslational modification occurs on the gp160 precursor glycoprotein can serve as an invaluable tool 


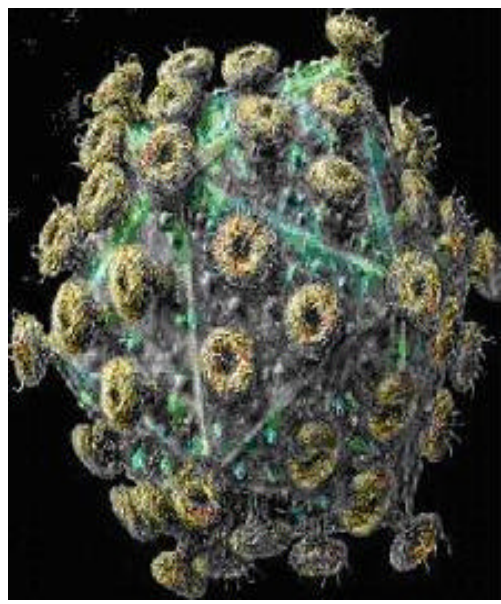

A

Figure 2. Structure of HIV

a) Structural view of the viral envelope $(0.1 \mu \mathrm{m}$ in diameter). Embedded in the envelope is a protein that consists of an outer protruding cap (gp120) and a stem (gp41) (10) . b) Close up view of the structure of gp41(11). c) X-ray crystallography image of gp120. Here, two views of the gp120 show the total topography of the glycoprotein. In white and yellow are the glycosylation sites(12).

for terminating the spread of the virus within the host.

Glycosylation of gp160 is extensive. The gp120 portion of gp160 has approximately 24 potential $\mathrm{N}$ linked glycosylation sites (Asn-X-Thr/Ser) (7) and the weight of the glycans make up over $50 \%$ of the molecular mass of the gp120 glycoprotein $(6,8)$ (figure 2 ). In contrast, the gp41 ectodomain is poorly glycosylated, with only four to five potential sites for $\mathrm{N}$-linked glycosylation (6). No evidence for O-linked glycosylation of the envelope proteins has been observed (9).

To study N-linked oligosaccharide structures on the HIV gp120, Mizouchi et al. used chronically HIVinfected lymphoblastoid (H9) cells and sequenced the glycans after they were released from proteins by hydrazinolosis. The conclusion from these studies is that the number of possible oligosaccharide structures present on gp120 outnumbers the 24 potential glycosylation sites present on the glycoprotein. Therefore, alternative structures occur on some of the glycosylation sites and numerous glycosylation variants of the gp120 are produced even in one cell line (13). The varied glycosylation is beneficial for the virus because it allows the virus to escape the immune system's response to the original invading virus by always appearing to the immune system as a new pathogen.

The importance of glycosylation sites in the infectivity of the virus has been investigated in both gp120 and gp41. This has been achieved by mutating the DNA sequences that code for the portion of glycoprotein important in its interaction with CD4 molecules on host cells. The results indicate that multiple mutations of glycosylation sites are required to alter the function of the gp120/gp41 complex (6). For example, the complete removal of glycan clusters from gp41 accomplished by altering the asparagines of the $\mathrm{N}$-linked glycosylation sequons in positions 621,630 , and 642 to serine residues, abrogated the viral ability to carry out fusion completely (6). Mutated gp41 comigrated with deglycosylated forms of wild-type gp41 on electrophoretic gels (14), suggesting that the deletion of the glycosylation sites contributes to the lack of glycosylation of the mutated proteins. These studies prove that for a successful HIV infection, the viral envelope proteins must be properly glycosylated so that the folding of the glycoproteins is correct and so that gp120 can interact with a CD4 host membrane receptor and allow the subsequent entry of the virus into the host cell. If gp41 is incompletely glycosylated, membrane fusion between the viral envelope and the host plasma membrane cannot occur (14). This finding may be useful in the construction of drugs that focus on the alteration of the oligosaccharide part of the HIV glycoprotein structure during its processing, decreasing the number of HIV particles that are capable of infecting CD4+ cells.

Additional studies involving mutated forms of the gp41 portion of gp160 have demonstrated that the transport of mutated forms of gp160 from the cis to the medial Golgi is slow and the transport to the trans Golgi is impaired. Therefore, since cleavage occurs in the trans part of the Golgi, cleavage reactions were severely impaired in the mutated gp160 at the gp120gp41 junction. Gp160 lacking gp41 carbohydrates demonstrated that proteins lacking glycans are arrested in the Golgi following biosynthesis. Therefore, the glycan components of the glycoprotein are important in 
the intracellular transport and processing of the gp160 (5).

$\mathrm{N}$-linked carbohydrates within the epitope portion of the HIV gp120 are important in the recognition of the HIV CD4 receptor. Support for this comes from a study by Botarelli et al. who immunized T-cells with a recombinant non-glycosylated gp120. When the immunized cells were presented with a wild-type glycosylated form of gp120, the cells' T-cell receptor did not recognize the glycosylated gp120 and did not develop a rapid immune response to it. To investigate the reason for the slow recognition of glycosylated glycoprotein, the epitope portion of the gp 120 bound to the T-cell was mapped. It was found that the epitope region of gp120 contained two asparagines residues which are sites for N-linked glycosylation (8). Therefore, the carbohydrates on the wild-type gp120 prevented epitope recognition by the immunized cells that had a T-cell receptor that was specific for the glycosylated form of gp120 used in the immunization.

The use of oligosaccharide processing inhibitors to study HIV glycoprotein structure

Previous studies have relied on utilizing glycobiology tools to better understand the mechanism of HIV infectivity and the source of its immunogenicity. For example, $\mathrm{N}$-linked glycosylation processing inhibitors can be used for studying the importance of protein glycosylation in HIV pathogenesis, replication, targetcell infectivity, and syncytium formation (15). The effect of oligosaccharide processing inhibitors can be determined by electrophoretic mobility. Proteins with the lowest molecular weight travel the furthest on the electrophoretic gel. In studies where gp120 is processed in the presence of glucosidase inhibitors (castanospermine or 1-deoxynojirimycin), there is a decrease in gp120 mobility. However, when mannosidase inhibitors (1-deoxymannojirimycin or swainsonine) are present, glycoprotein mobility is increased (15). These results suggest that the final glycan products found on gp120 are smaller that the glycan products seen when glucosidase inhibitors are added and bigger than high mannose chains that are seen when mannosidase inhibitors are introduced into the media.

Inhibitors of oligosaccharide processing can also be used to study the steps in the processing of envelope glycoproteins in cells. For example, treating chronically HIV-infected cells with tunicamycin (an inhibitor that completely abrogates the addition of glycans to N-linked glcosylation sites of the protein) severely inhibits the glycosylation of envelope proteins. Treatment with deoxynojirimycin, an inhibitor of glucosidase $I$ in the rough ER, inhibits proteolytic cleavage of gp160 $(9,15)$. Inhibitors of mannosidase I and mannosidase II (deoxymannojirimycin and swainsonine respectively) allow for the processing of the gp160 to gp120 and gp41. However, the virions made by cells treated with mannosidase inhibitors are significantly less infectious than virions synthesized by untreated cells. This suggests that proteolytic cleavage of gp160 takes place in infected cells when the glycoprotein has mannose-rich oligosaccharide structures. However, for the release of infections virions, the trimming of glucose residues and the primary trimming of mannose are both necessary (15). Therefore, oligosaccharide processing inhibitors can be used to decrease the infectivity of new HIV virions since the viral particles would contain improperly glycosylated glycoproteins.

\section{HIV THERAPY BASED ON CURRENT KNOWLEDGE OF GLYCOBIOLOGY}

HIV is a very complicated pathogen due to its ability to mutate at a fast rate. It introduces difficulty for the immune system, which continually sees the virus as foreign, posing challenges to treatment. Research in the field of HIV has contributed to a wealth of therapeutic options. Current treatments can be classified into the following classes: (i) nucleoside/nucleotide reverse transcriptase inhibitors (NRTIs) (i.e. zidovudine (AZT), (ii) non-nucleoside reverse transcriptase inhibitors (NNRTIs) (i.e. nevirapine); and (iii) protease inhibitors (PIs) (i.e. saquinavir). Other steps in the HIV replicative cycle that are potential targets for chemotherapeutic intervention include viral adsorption, viral entry, virus-cell fusion, proviral DNA integration, and viral mRNA transcription (16). Additionally, the expanding field of glycobiology proposes additional treatment options.

HIV drug therapy based on inhibitors of oligosaccharide processing

Application of carbohydrate analogues such as tunicamycin (inhibitor of the synthesis of the dolichollinked oligosaccharide precursor), castanospermine and 1-deoxynojirimycin (DMN, glucosidase inhibitor), as well as 1-deoxymannojirimycin (mannosidase I inhibitor), has been shown to attenuate the HIV-1 infectivity invitro. These inhibitors oligosaccharide processing are ineffective in preventing the formation of a cytoplasmic continuity (syncytia) between two cells when gp120/gp41 complexes are targeted to the infected cell's plasma membrane and bind to CD4 receptors of neighboring CD4+ cells (15). However, Nglycosylation is critical in HIV pathogenesis at the level of viral binding and fusion to uninfected CD4+ cells.

The gp120/gp41 HIV envelope complex must be 

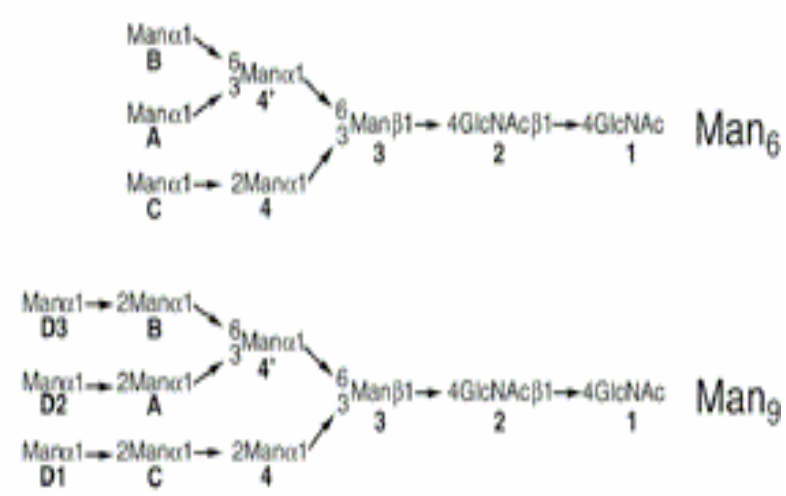

Figure 3. Formulas of $\mathrm{N}$-linked oligomannose molecules including $\operatorname{Man}(6)$ and $\operatorname{Man}(9)$. Isomers of $\operatorname{Man}(7)$ and $\operatorname{Man}(8)$ can be deduced by adding mannose termini to the Man(6) structure (18).

folded properly for it to be pathogenic. Proper folding is mediated by oligosaccharides on the gp120/gp41 complex. Therefore, glycosylation inhibitors can be applied to alter the fusogenicity of the gp120/gp41 complex since improperly folded proteins cannot induce fusion. For example, DNM inhibits the cellular a-glucosidase I-II activity, blocking the trimming of the glycan precursor at the level of the ER (i.e. the cleavage of three glucose residues from $\operatorname{Glc}(3) \operatorname{Man}(9) \operatorname{GlcNAc}(2)$ precursor glycan, see figure 3). In the presence of DNM, glycoproteins with abnormally glycosylated oligomannosidic moieties are produced. Treatment of HIV-infected lymphocyte cultures with DNM inhibited the spread of the virus (16). This was possible because although the gp120/pg41 complex synthesized in the presence of DNM could bind CD4 receptors on human lymphocytes, it was not able to induce membrane fusion. N-butyl DNM, an alkyl substituted variant of DNM, has shown antiviral effects at doses with no reported cell cytotoxicity (3). Phase I clinical trials on n-butyl DNM in twenty-nine patients, however, showed no significant trends in CD4+ cell counts, and Grade II elevations in liver function, leucopenia, and neutropenia were observed in some patients, calling the study to a halt before the dose-escalading tolerance trial could be completed (17).

\section{HIV drug therapy based on oligosaccharide structure}

If the glycan structure composition of the HIV glycoprotein gp120 can be determined, the interaction between gp120 and CD4+ cell can become a potentially useful target in HIV therapy. To study the gp120 epitopes involved in interaction with the CD4 receptor, three $\mathrm{N}$-linked glycosylation sites on the gp120 in the vicinity of the epitope recognized by the host T-cell were removed (7). Mutant gp120 with one, two, and three $\mathrm{N}$-linked glycans missing were analyzed. The conclusions made were that two of the three glycosylation sites were heterogeneous in the structural composition of glycans. However, the site located next to the T-cell epitope had a large, high mannose structure with more than 11 mannose units and it covered a large part of the surface of gp120 (7). The finding that high mannose glycan structure present in the epitope of the gp120 is recognized by the T-cell has allowed for the creation of cyanovirin-N (CV-N), a drug that inactivates diverse strains of HIV at the level of cell fusion by virtue of its interaction with specific N-linked oligosaccharides on gp120.

$\mathrm{CV}-\mathrm{N}$ is a $11 \mathrm{kDa}$ monomeric protein derived from cyanobacteria. CV-N binds to glycosylated gp120 but not to unglycosylated gp120. When the ligand which binds to CV-N was studied by examining the binding of gp120 to a CV-N affinity column, only Man-8 and Man9 glycoforms (figure 3) were preferentially retained on the affinity column. Studies have found that adding free Man-8 and Man-9 oligosaccharides partially inhibited binding of CV-N to gp120 (2). When a mixture of carbohydrates that structurally represent $\mathrm{N}$-linked carbohydrates found on the gp120 was added, it was found that CV-N specifically recognizes with nanomolar affinity $\operatorname{Man}(9) \operatorname{GlcNAc}(2)$ and Man(8)GlcNAc(2) (18). Viruses sensitive to CV-N exhibit an abundant exposure of high mannose oligosaccharides on their surfaces (figure 3) (19). All of the above findings suggest that the part of gp 120 which is recognized by $\mathrm{CV}-\mathrm{N}$ is the high mannose glycan portion of the glycosylated gp 120 .

$\mathrm{CV}-\mathrm{N}$ is unlike other lectins that have been reported to have anti-HIV activity mediated through interactions with gp120. Classical carbohydrate-interacting lectins, such as concanavalin A and wheat-germ agglutinin, associate with gp120 in a monosaccharide-specific manner and are inhibited by the presence of exogenous monosaccharides. However, CV-N/gp120 interaction is not inhibited by high concentrations (i.e. 10,000-fold excess) of monosaccharides (19). This observation suggests that CV-N/gp120 interactions are defined by a more complex oligosaccharide-specific binding. In addition, the carbohydrate structures recognized by CV$\mathrm{N}$ are rare in normal human tissue. Therefore, $\mathrm{CV}-\mathrm{N}$ is a potential oligosaccharide-specific therapeutic agent for the treatment of HIV as well as other pathogens with high mannose ligands on their outer surface.

Besides CV-N, other drugs have also been designed based on the principle that high mannose chains are essential for HIV infection. For example, Pradmicin A, an antifungal antibiotic isolated from Actnomdura hibisca, and its derivative, BMY-28864, have the ability to inhibit HIV infection in vitro (20). Similar to the CV$\mathrm{N}$ studies, the inhibitory effects of these drugs were suppressed by the addition of high mannose type 
oligosaccharides. Therefore, targeting oligosaccharide chains of the envelope glycoprotein is a possible way to block HIV infection.

\section{Therapy based on oligosaccharide structure to construct a potent HIV vaccine}

An effective way of combating HIV is via vaccination. To construct a successful vaccine, the HIV gp120 antigen needs to be recognized by the helper Tcell. In order to generate vaccines that will induce a strong immune response, the HIV gp120 antigen needs to be made more foreign. In one vaccination study, gp120 was made more immunogenic by neuraminidase treatment, which removed terminal salicylic acids from the carbohydrate side-chains of the glycoprotein, producing an asialoglycoprotein (21). Neuraminidase treatment exposed terminal galactose residues so that they could be recognized by galactose receptors on antigen presenting cells. Evidence for the use of galactose receptors became apparent when galactose is added to cultures of antigen presenting cells. Extrinsic galactose, when added, competes with asialoglycoproteins for binding to galactose receptors. Thus, the antigenicity of the HIV glycoprotein gp120 can be enhanced by exposing galactose residues on the gp120 since these glycoproteins can be internalized by antigen presenting cells containing galactose receptor. Uptake of the proteins with terminal galactose units results in a quicker immune response because asialoglycoproteins are more quickly recognized as foreign (4).

When constructing an HIV vaccine, it is therefore worthy to explore glycobiology to produce a vaccine with a more immunogenic antigen. If a more antigenic form of gp120 could be administered to a patient, the patient may be able to mount a stronger immune response against the HIV.

\section{Inhibition of HIV gp120 binding to CD4 molecules by natural glycosaminoglycans}

It is a fact that HIV is spread via body fluids. Past studies of HIV inhibitors have suggested that the presence of inhibitory macromolecules in the body fluids that do not transmit the virus could be responsible for the inability for these fluids to function as a vehicle for HIV transmission. Other body fluids that serve as a route of HIV transmission, such as breast milk, have been found to contain compounds that could limit the rate of postnatal vertical transmission for HIV in breastfed infants of HIV-infected mothers. The proposed reason for the lack of viral transmission by some body fluids is the presence of glycosaminoglycans (22).

Previous studies have proven that non-antibody glycoconjugates from human milk can inhibit the binding of many pathogenic microorganisms.
According to Newburg et al, glycosaminoglycans found in human milk represent a novel class of naturally occurring molecules that are capable of inhibiting viral binding to host CD4 receptors (22).

Glycosaminoglycans are heteropolysaccharides; they are linear polymers made of repeating disaccharide units. One of the monosaccharides is always Nacetylglucosamine or $\mathrm{N}$-acetylgalactosamine, and the other is a uronic acid such as glucuronic acid. One or more of the hydroxy groups of the amino sugars can be sulfonated (4), producing glycosaminoglycans such as dermatan sulfate, heparan sulfate, or chondroitin sulfate.

Sulfonated molecules, including sulfonated glycosaminoglycans (GAG), have been shown to inhibit the binding and replication of HIV. The classes of GAG found in human milk include heparin, heparan sulfate, chondroitin sulfate, and dermatan sulfate .

To determine the types of glycosaminoglycans that are most important in the inhibition of HIV replication, Newburg et al analyzed milk from thirty women. To test for the inhibitory nature of each glycosaminoglycan from human milk, isolated GAGs, CD4 and gp120 molecules were mixed and allowed to interact. Glycosaminoglycan cleaving enzymes (lyases) were then added to cleave the linear glycosaminoglycan polymeric backbone and the reaction mixtures were tested for the loss of inhibitory action. The greatest loss in the inhibition of the gp120 and CD4 interaction was seen when chondroitinase AC was added (22). This suggests that the specific glycosaminoglycan component of human milk responsible for the inhibition of HIV binding and replication is chondroitin sulfate.

Further research on sulfated glycosaminoglycans could provide valuable treatment methodologies to terminate the HIV epidemic. Based on the studies of sulfated glycosaminoglycans, synthetic sulfonated polymers that share the gross features of GAGs could be synthesized such that they contain anti-HIV activity.

\section{CONCLUSION}

New antiretroviral therapies prolong the lifespan of people infected with HIV. Correspondingly, the number of AIDS-related deaths has declined. Despite the availability of drug therapies, HIV is still a challenge. Glycobiology is a field with foreseeable therapeutic alternatives for HIV. Glycobiology-related treatments such as inhibitors of oligosaccharide processing, treatments based on blocking the reactive sites of gp120, vaccinations, and natural methods using glycosaminoglycans to combat the deadly virus, further add to the list of potential antiretroviral treatments. Combining these treatments with current antiretroviral methods could result in synergistic anti-HIV effects. 
However, further testing of the glycobiology-related therapies is needed to determine the non-specific effects on other cellular processes and before the therapies can be made available for the HIV-affected population.

\section{REFERENCES}

1. International AIDS Vaccine Initiative. http://iavi.org ; 2002.

2. Bloom, DE. Something to be done: treating HIV/AIDS. River Path Associates. Science 288(5474):2171-3; 2000.

3. Ratner L. Glucosidase Inhibitors for Treatment of HIV-1 Infection. Aids Research and Human Retroviruses 8:165-173; 1992.

4. Lehninger Albert L, Nelson David L, Cox Michael M. Principles of Biochemistry, 2nd edition. New York, NY: Worth Publishers, 1993.

5. Gilbert PB, McKeague IW, Eisen G, Mullins C, Gueye-NDiaye A, Mboup S, Kanke PJ. Comparison of HIV-1 and HIV-2 infectivity from a prospective cohort study in Senegal. Statist. Med 22:573-593; 2003.

6. Pal R, Hoke, GM, Sarngadharan MG. Role of gp41 Glycosylation Sites in the Biological Activity of Human Immunodeficiency Virus Type 1 Envelope Glycoprotein. Virology 242:338-345; 1998.

7. Biller M, Bolmstedt A, Hemming A, Olofsson S. Simplified procedure for fractionation and structural characterization of complex mixtures of N-linked glycans, released from HIV-1 gp120 and other highly glycosylated viral proteins. J Virol Methods 76:87-100; 1998.

8. Botarelli P, Houlden BA, Haigwood NL, Servis C, Montagna D, Abrignani S. N-glycosylation of HIV-gp120 may constrain recognition by T lymphocytes. J Immunol 147:3128-3132; 1991.

9. Kozarsky K, Penman M, Basiripour L, Haseltine W, Sodroski J, Krieger M. Glycosylation and processing of the human immunodeficiency virus type 1 envelope protein. J Acquir Immune Defic Syndr 2:163-169; 1989.

10. AVERT, AIDS Virus Education and Research Trust. www.avert.org/virus.htm ; 2002.

11. Howard Hughes Medical Institute. Key HIV Protein Structure Solved. http://www.hhmi.org/news/keyhiv.html ; 1997.

12. Zhu X, Borchers C, Bienstock RJ, Tomer KB. Mass Spectrometric Characterization of the Glycosylation Pattern of HIV-gp120 Expressed in $\mathrm{CHO}$ Cells. Biochemistry 39:11195;11204; 2000.
13. Mizuochi T, Matthews TJ, Kato M., Hamako J, Titani K, Solomon J, Feizi T. Diversity of oligosaccharide structures on the envelope glycoprotein gp120 of human immunodeficiency virus 1 from the lymphoblastoid cell line H9. Presence of complex-type oligosaccharides with bisecting $\mathrm{N}$ acetylglucosamine residues. J Biol Chem 265:8519-8524; 1990.

14. Fenouillet E, Jones I, Powell B, Schmitt D, Kieny MP, Gluckman JC. Functional role of the glycan cluster of the human immunodeficiency virus type 1 transmembrane glycoprotein (gp41) ectodomain. J Viro, 67:150-160; 1993.

15. Pal R, Hoke GM, Sarngadharan MG. The role of oligosaccharides in the processing and maturation of envelope glycoproteins of human immunodeficiency virus type 1 . Proc Natl Acad Sci USA 86:3384-3388; 1989.

16. De Clercq E. New developments in anti-HIV chemotherapy. Curr Med Chem 8:1543-72; 2001.

17. Tierney M, Pottage J, Kessler H, et al. The tolerability and pharmacokinetics of N-butyl-deoxynojirimycin in patients with advanced HIV disease (ACTG 100). The AIDS Clinical Trials Group (ACTG) of the National Institute of Allergy and Infectious Diseases. J Acquir Immune Defic Syndr Hum Retrovirol 10:549-553; 1995.

18. Bewley CA, Otero-Quintero S. The potent anti-HIV protein cyanovirin- $\mathrm{N}$ contains two novel carbohydrate binding sites that selectively bind to $\operatorname{Man}(8)$ D1D3 and Man(9) with nanomolar affinity: implications for binding to the HIV envelope protein gp120. J Am Chem Soc 123:38923-902; 2001.

19. Bolmstedt AJ, O'Keefe BR, Shenoy SR McMahon JB, Boyd MR. Cyanovirin- $N$ defines a new class of antiviral agent targeting N-linked, high-mannose glycans in an oligosaccharide-specific manner. Mol Pharmacol 59:949-954; 2001.

20. Mizuochi T, Nakata M. New anti-HIV drug which binds the oligosaccharides of HIV envelope glycoprotein. Nippon Rinsho 53:2340-2349;1995.

21. Manca F. Galactose receptors and presentation of HIV envelope glycoprotein to specific human T cells. J Immunol 148:22782282; 1992.

22. Newburg DS, Linhardt RJ, Ampofo SA, Yolken RH Human Milk Glycosaminoglycans Inhibit HIV Glycoprotein gp120 Binding to Its Host Cell CD4 Receptor. J Ntr 125:419-423; 1995.

23. Medical microbiology, Glycosaminoglycans. http://www.indstate.edu/thcme/mwking/glycans.html ; 2001.

Aleksandra M. Leligdowicz is a second year medical student at the McGill University Faculty of Medicine. In 2000, she completed a BSc degree in molecular biology at the University of Manitoba and Deakin University, Australia. In October 2004, she will begin research in Immunology and Epidimiology of HIV-2 at the Institute of Molecular Medicine at Oxford University as a Rhodes Scholar. 